\title{
Oral Condition Between Assisted Reproductive Technology Children And Natural Conceived Children
}

Norzaiti Mohd Kenali ${ }^{1}$, Mohd Syazwan Aidid Mohamad Basir ${ }^{2}$, Mohd Firdaus Lokhman $^{2}$, Susi Sukmasari ${ }^{1}$, Roszaman Ramli ${ }^{3}$

${ }^{1}$ Paediatric Dentistry Unit, Kulliyyah of Dentistry, International Islamic University Malaysia

${ }^{2}$ Kulliyyah of Dentistry, International Islamic University Malaysia

${ }^{3}$ Department of Obstetric and Gynaecology, Kulliyyah of Medicine, International Islamic University Malaysia

Presenter: Norzaiti Mohd Kenali

Introduction: Assisted Reproductive Technology (ART) children were shown to have higher neurological and musculoskeletal disorder. However, limited number of studies found concerning oral condition of ART children. This research aimed to determine oral condition between ART children and Natural Conceived (NC) children. Materials and Methods: This cross-sectional study consisted of 34 subjects, 17 ART children and 17 NC children. Intraoral examination and orthopantomogram (OPG) radiograph were done in these subjects. Any abnormalities of tooth development such as number and tooth structure was recorded. Caries experience of both groups were recorded using decayed, missing, filled tooth (dmft) index. Descriptive analysis and Mann-Whitney's Test were used. Results: Most of the participants were female (58.8\%) and majority aged 7 to 8 years old $(47.1 \%)$. Of the patients, $70.6 \%$ of ART group were from intra-uterine insemination (IUI) technique while $29.4 \%$ were from in-vitro fertilization (IVF). One hypodontia case were found in each group. Caries experience in primary teeth were significantly lower in the ART group compared the NC group, with $p$ value of 0.003. Conclusion: Children in ART group experience less dental caries in the primary teeth compared with NC children. However, there was a comparable case of tooth developmental defect in both groups. 